\title{
Self-Assembled Nanoparticles of Dextrin Substituted with Hexadecanethiol
}

\author{
Catarina Gonçalves, ${ }^{\dagger}$ José A. Martins,${ }^{\ddagger}$ and Francisco M. Gama ${ }^{*, \dagger}$ \\ Centro de Engenharia Biologica and Departamento de Química, Universidade do Minho, Campus de \\ Gualtar, 4710-057 Braga, Portugal
}

Received October 16, 2006

\begin{abstract}
The amphiphilic molecule dextrin-VA-SC $\mathrm{S}_{16}\left(\mathrm{dexC}_{16}\right)$ was synthesized and studied in this work. $\mathrm{DexC}_{16}$ has a hydrophilic dextrin backbone with grafted acrylate groups (VA) substituted with hydrophobic 1-hexadecanethiol $\left(\mathrm{C}_{16}\right)$. A versatile synthetic method was developed allowing control of the dextrin degree of substitution with the hydrophobic chains $\left(\mathrm{DS}_{\mathrm{C} 16}\right.$, number of alkyl chains per 100 dextrin glucopyranoside residues). Materials with different $\mathrm{DS}_{\mathrm{C} 16}$ were prepared and characterized using ${ }^{1} \mathrm{H}$ NMR. DexC $\mathrm{C}_{16}$ self-assembles in water through association of the hydrophobic alkyl chains, originating nanoparticles. The nanoparticles properties were studied by dynamic light scattering (DLS), fluorescence spectroscopy, and atomic force microscopy (AFM).
\end{abstract}

\section{Introduction}

Amphiphilic molecules, such as surfactants or lipids, spontaneously self-assemble in water, forming self-aggregates, such as micelles, bilayer membranes, tubes, and vesicles. Amphiphilicity of biopolymers is one of the most important factors for their self-organization in water. Among the different types of amphiphilic polymers, water-soluble polymers with hydrophobic molecules grafted on side chains have received special attention. By self-assembling, the hydrophobic segments are segregated from the aqueous exterior to form an inner core surrounded by hydrophilic chains. Polymeric micelles or nanoparticles with a hydrophobic core and hydrophilic shell are thus prepared. This kind of structure is suitable for trapping hydrophobic substances, such as fluorescent probes, ${ }^{1}$ proteins,${ }^{2}$ and hydrophobic pharmaceuticals. ${ }^{3}$ The size, density, and colloidal stability of nanoparticles can be controlled by changing the degree of substitution of hydrophobes and its hydrophobicity. ${ }^{4}$ The association mechanism is mainly governed by the alkyl side chain concentration and length and is little influenced by the molecular weight of the polymer backbone. ${ }^{5}$ However, with low molecular weight polymers, the hydrophobic aggregates are not connected via the polymer backbone. ${ }^{6}$ The study of nanogels (hydrogel nanoparticles) has intensified during the past decade due to enormous potential applications in the development and implementation of new environmentally responsive materials, biomimetics, biosensors, artificial muscles, and drug delivery systems. ${ }^{7}$ Solid nanoparticles made from biodegradable polymers have been widely investigated for long-term delivery of drugs. ${ }^{8}$ They can potentially provide benefits such as increased therapeutic effect, prolonged bioactivity, controlled release rate, and finally decreased administration frequency, thereby increasing patient compliance.

Nanostructures spontaneously form when the concentration of the polymer is higher than its critical micelle concentration (cmc). Self-assembly in water of a hydrophobically modified dextrin $\left(\operatorname{dex}_{16}\right)$ was investigated in this work. The structural change upon dilution of the $\operatorname{dex}_{16}$ self-aggregates in water was investigated by fluorescence in the presence of pyrene as the

\footnotetext{
Centro de Engenharia Biológica.

$\doteqdot$ Departamento de Química.
}

Table 1. Dextrin Degree of Substitution Obtained Using Different 1-Hexadecanethiol/VA Molar Ratio

\begin{tabular}{|c|c|c|}
\hline theoretical DSa (\%) & obtained DS ${ }^{b}(\%)$ & efficiency $^{c}(\%)$ \\
\hline 10 & 2 & 20 \\
\hline 20 & 4 & 20 \\
\hline 40 & 14 & 35 \\
\hline 60 & 23 & 38 \\
\hline 100 & 59 & 59 \\
\hline
\end{tabular}

${ }^{a}$ Calculated as the molar ratio of 1-hexadecanethiol to acrylate groups $(\times 100)$ in the reaction mixture. ${ }^{b}$ Determined by ${ }^{1} \mathrm{H}$ NMR. The value shown is the calculated using eq 3 , multiplied by 5 , to obtain the DS relative to acrylate groups. ${ }^{c}$ Calculated as the ratio of the obtained to the theoretical DS $(\times 100)$.

fluorescent probe. Pyrene shows interesting photophysical properties due to the long lifetime of its monomers. Pyrene is one of the few condensed aromatic hydrocarbons which shows significant fine structure (vibronic bands) in its monomer fluorescence spectra in solution. The vibrational fine structure intensities undergo significant perturbations on going from nonpolar solvents to polar solvents with high permanent dipoles. The five predominant peaks of pyrene are numbered $\mathrm{I}-\mathrm{V}$, and peak III shows maximum variations in intensity relative to peak I. The $I_{3} / I_{1}$ ratio will be used for determination of the critical micelle concentration. ${ }^{9}$ The cmc value depends mostly on the chemical structure of the amphiphilic polymer and the number of hydrophobic chains grafted on the polymer backbone (DS). Other relevant properties of the nanoparticles, such as the size, stability, and shape, were also evaluated in this work.

\section{Experimental Section}

Materials. Dextrin-VA was synthesized by transesterification of dextrin with vinyl acrylate (VA) as described by Ferreira et al. ${ }^{10}$ for the transesterification of dextran with VA with few modifications. The transesterification of dextran requires an enzyme (Proleather), but using dextrin as substrate the enzyme does not catalyze the transesterification. In this work, dextrin-VA with 20 acrylate groups per 100 dextrin glucopyranoside residues (DS $\mathrm{VA}_{\mathrm{A}} 20 \%$ ) was used. Dimethyl sulfoxide (DMSO), triethylamine (TEA), and deuterium oxide $\left(\mathrm{D}_{2} \mathrm{O}\right)$ were from Aldrich. Regenerated cellulose tubular membranes, with 3500 MWCO, were obtained from Membrane Filtration Products. 
Scheme 1. Synthesis of $\operatorname{DexC}_{16}$
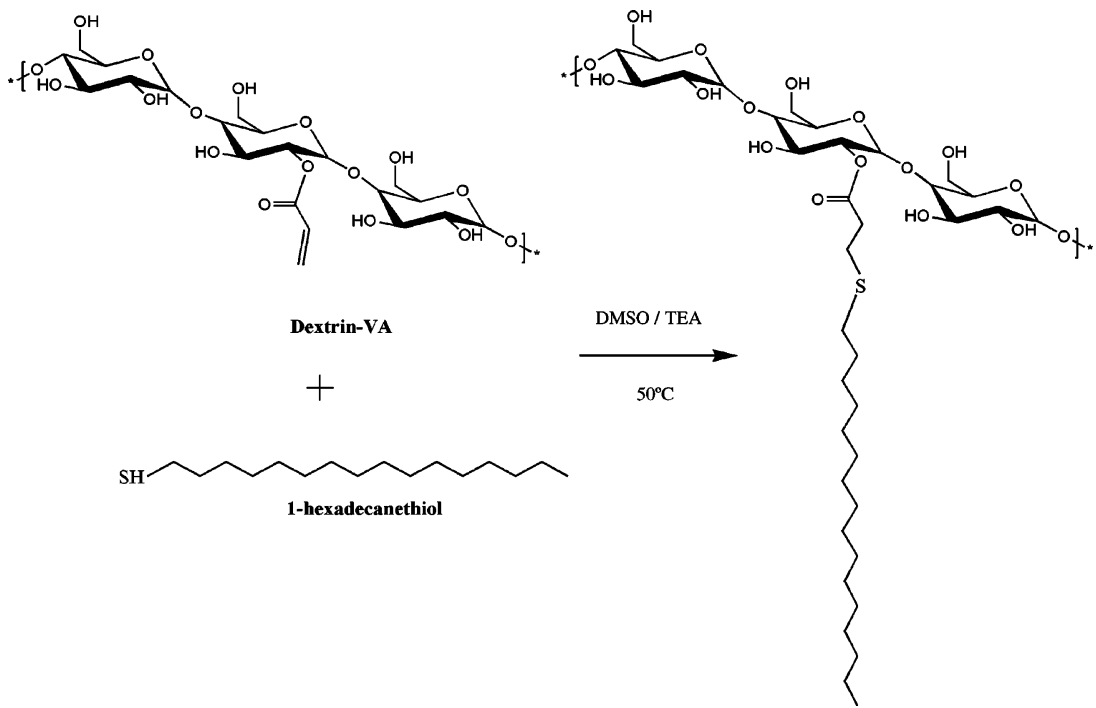

Pyrene (Py) was from Aldrich and used after appropriate recrystallization. Distilled water was used for the preparation of aqueous solutions.

Synthesis of DexC $C_{16}$. Dextrin-VA and 1-hexadecanethiol were dissolved in dimethyl sulfoxide (equivalent VA $=0.0413 \mathrm{M}$ ). Different molar percentages of 1-hexadecanethiol $(10 \%, 20 \%, 40 \%, 60 \%$, and $100 \%$ relative to VA) were added to the reaction mixture in order to obtain different levels of grafting. Triethylamine (1 mol equiv to VA) was added to the reaction mixture. The medium was stirred for $24 \mathrm{~h}$ at $50{ }^{\circ} \mathrm{C}$. The mixture was dialyzed for $48 \mathrm{~h}$ against water with frequent water change. After freezing, the mixture was lyophilized and stored.

Sample Preparation. Lyophilized $\operatorname{dex} \mathrm{C}_{16}$ was dissolved in water under stirring at $50{ }^{\circ} \mathrm{C}$ and then further sonicated for 20 min until a clear solution was obtained. The degree of solubility of $\operatorname{dexC}_{16}$ depends on the degree of substitution. Increasing the degree of substitution reduces the solubility. In the range of $\mathrm{DS}_{\mathrm{C} 16}$ used, to prepare a $1 \mathrm{~g} / \mathrm{dL}$ solution, $3 \mathrm{~h}$ of stirring is the maximum time required to dissolve dexC $\mathrm{C}_{16}$.

Dynamic Light Scattering (DLS). The size distribution was determined with a Malvern Zetasizer, NANO ZS (Malvern Instruments Limited, U.K.). A dispersion of nanoparticles in ultrapure water $(1 \mathrm{~mL})$ was analyzed at $25^{\circ} \mathrm{C}$ in a polystyrene cell using a $\mathrm{He}-\mathrm{Ne}$ laserwavelength of $633 \mathrm{~nm}$ and detector angle of $173^{\circ}$. The dispersion was filtered through a $0.45 \mu \mathrm{m}$ pore.

${ }^{1} \mathbf{H}$ NMR. Lyophilized dex $\mathrm{C}_{16}$ was dispersed in deuterium oxide (10 $\mathrm{mg} / \mathrm{mL}$ ). Solutions were transferred to $5 \mathrm{~mm}$ NMR tubes. 1D ${ }^{1} \mathrm{H}$ NMR measurements were performed with a Varian Unity Plus 300 spectrometer operating at $299.94 \mathrm{MHz}$. 1D ${ }^{1} \mathrm{H}$ NMR spectra were measured at $298 \mathrm{~K}$ with 80 scans, a spectral width of $4800 \mathrm{~Hz}$, a relaxation delay of $1 \mathrm{~s}$ between scans, and an acquisition time of $3.75 \mathrm{~s}$.

Fluorescence Spectroscopy. Fluorescence measurements were performed on a Varian Cary Eclipse fluorescence spectrofluorometer using a quartz cell. The pyrene spectra were obtained using an excitation wavelength of $337 \mathrm{~nm}$ and recording the emission over the range 350$500 \mathrm{~nm}$ at a scan rate of $120 \mathrm{~nm} / \mathrm{min}$. The slit width was set at $20 \mathrm{~nm}$ for the excitation and $2.5 \mathrm{~nm}$ for the emission. The fluorescence intensity, for each vibronic peak, was measured at the maximum of each peak.

Atomic Force Microscopy (AFM). Tapping-mode imaging was carried out on a Nanoscope IIIa Multimode (Digital Instruments, Veeco) scanning probe microscope. A silicon tip doped with phosphorus, with a radius curvature of less than $10 \mathrm{~nm}$ (RTESP, VEECO), was used. This tip has a typical resonance frequency of $288-328 \mathrm{kHz}$ and a typical force constant of $20-80 \mathrm{~N} / \mathrm{m}$. A scan rate of $1.4-1.8 \mathrm{~Hz}$ was sufficient to maintain a good signal-to-noise ratio. A drop of aqueous solution $(1 \mathrm{mg} / \mathrm{mL})$ of $\operatorname{dex}_{16}$ was placed on new-cleaved HOPG surface, thoroughly rinsed with water, and dried under a $\mathrm{N}_{2}$ flux.

\section{Results and Discussion}

Synthesis of the Dextrin-VA-SC 16 . The reaction between the thiol moiety and the acrylate group of dextrin-VA is a Michael addition with thiol acting as a nucleophile (Scheme 1).

${ }^{1} \mathrm{H}$ NMR was used to analyze the structure of the reaction product. The signals between 5.8 and $3.0 \mathrm{ppm}$ in the ${ }^{1} \mathrm{H}$ NMR spectrum of dextrin-VA are assigned to protons from the dextrin scaffold. The protons from the acrylate group, attached to the dextrin backbone are observed between 6.6 and $6.0 \mathrm{ppm},{ }^{10}$ as shown in Figure 1. The presence of two different positional isomers in dextrin-VA was revealed by the ${ }^{1} \mathrm{H}-{ }^{1} \mathrm{H}$ COSY and ${ }^{1} \mathrm{H}-{ }^{13} \mathrm{C}$ HMQC spectra. The two positional isomers are located at positions 2 and 3 in the glucopyranosyl residues (data not shown) in the main dextrin backbone. For the sake of simplicity, only the 2-O-acryloyl regioisomer is represented in Scheme 1.

The reaction between the grafted acrylate and the thiol hexadecanethiol follows a mechanism of Michael addition. The intensity of the signals from protons of the unsaturated carbons of the acrylate groups decreases as the reaction progresses, and the signals should eventually disappear completely when all acrylate groups are grafted with thiol moieties. Simultaneously, a new signal assigned to the methylene protons should appear. Preliminary experiments were carried out without a base catalyst. Although it was possible to identify the signals corresponding to the grafted alkyl moiety, between 2.0 and $0.6 \mathrm{ppm}$, the acrylate protons are still detected. Using TEA as a catalyst, the signals from thiol moieties have higher intensity and the acrylate signals completely disappear, confirming the success of the synthesis (Figure 2). Therefore, a base catalyst must be used for a high reaction yield to be obtained.

In order to ascertain whether the thiol is covalently bound, dexC $_{16}$ was washed several times with $n$-hexane. As no differences were detected in the ${ }^{1} \mathrm{H}$ NMR analysis, it may be concluded that the alkyl chain is covalently bound to acrylate.

DLS was also used to check the possibility of self-polymerization of acrylic groups at $50{ }^{\circ} \mathrm{C}$. The size distribution of dextrin-VA in DMSO was evaluated before and after stirring at $50{ }^{\circ} \mathrm{C}$ for $24 \mathrm{~h}$. No differences were observed; 


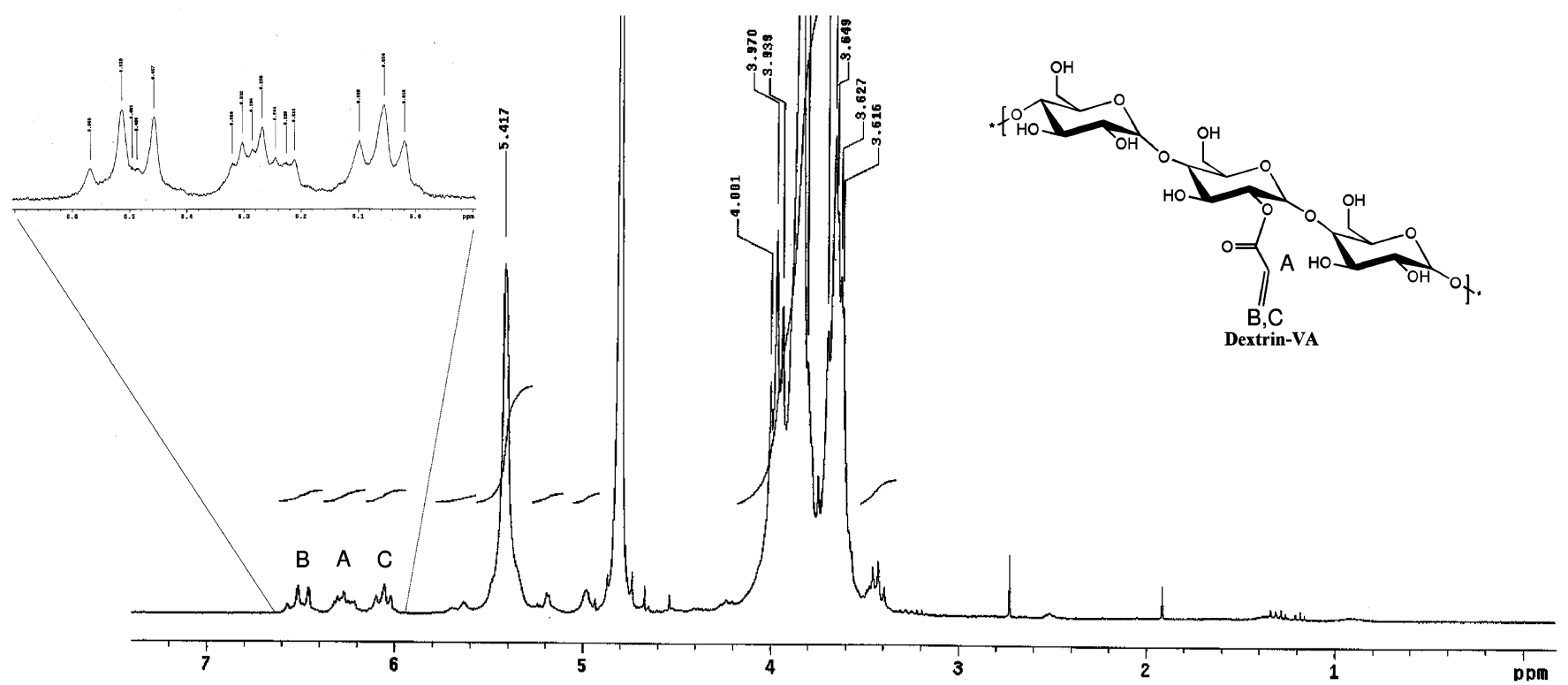

Figure 1. ${ }^{1} \mathrm{H}$ NMR spectra of dextrin-VA (DS $V_{V A} 20 \%$ ) in $\mathrm{D}_{2} \mathrm{O}$ at $25^{\circ} \mathrm{C}$.

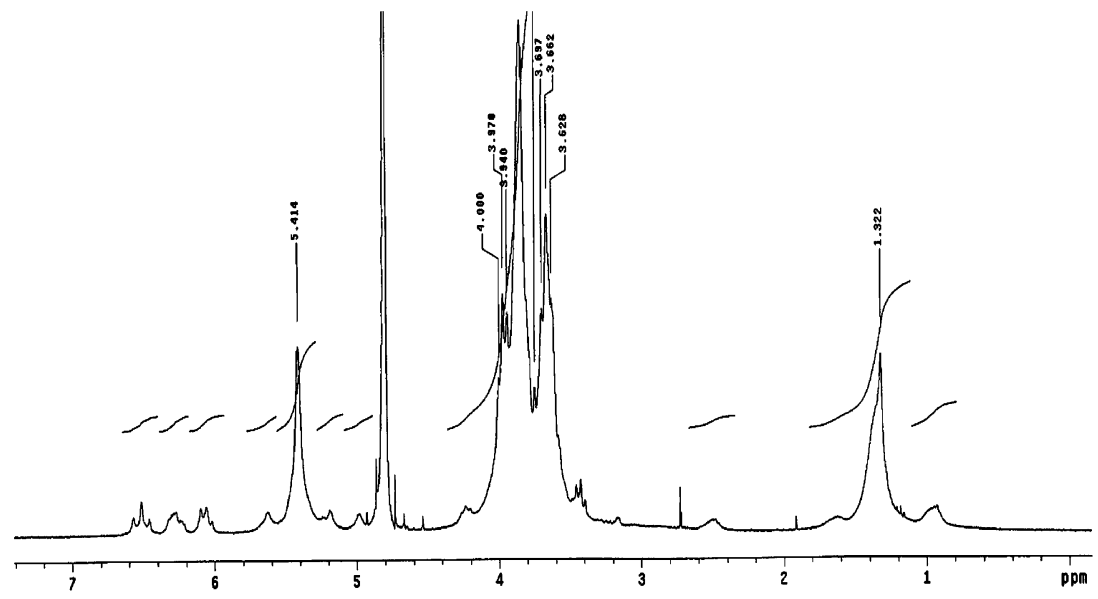

(a)

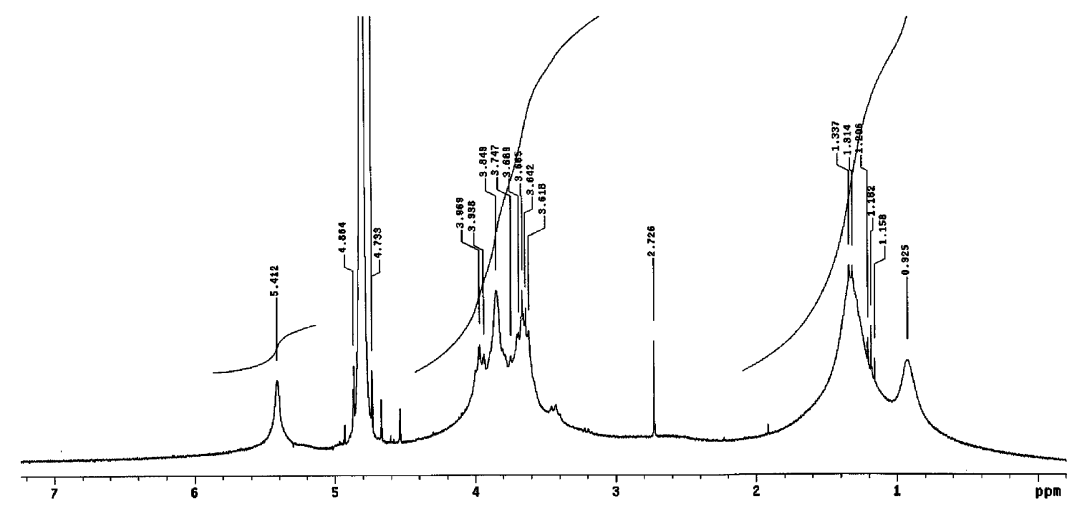

(b)

Figure 2. ${ }^{1} \mathrm{H}$ NMR spectra in $\mathrm{D}_{2} \mathrm{O}$ of dextrin-VA reacted with an excess of hexadecanethiol (a) without and (b) with TEA.

therefore, the polymerization does not take place during the synthesis reaction.

Degree of Substitution. The synthesis of $\operatorname{dex}_{16}$ with different degrees of substitution was accomplished by varying the molar ratio 1-hexadecanethiol/VA in the reaction mixture. The ${ }^{1} \mathrm{H}$ NMR spectrum of $\operatorname{dex} \mathrm{C}_{16}$, in deuterated water, was used to determine the degree of substitution obtained $\left(\mathrm{DS}_{\mathrm{C} 16}\right.$, amount of alkyl chains per 100 dextrin glucopyranoside residues). $\mathrm{DS}_{\mathrm{C} 16}$ was calculated as a peak area ratio in the NMR spectra according to eq 3

$$
\mathrm{DS}_{\mathrm{C} 16}=\frac{7 x}{37 y} \times 100
$$



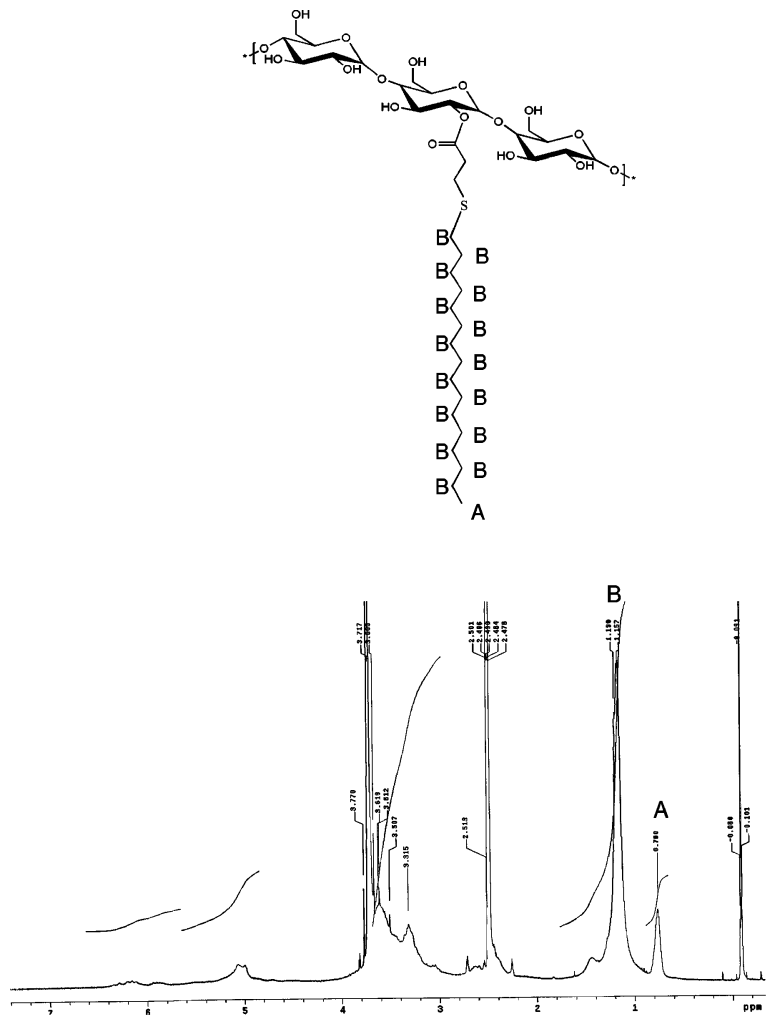

(b)

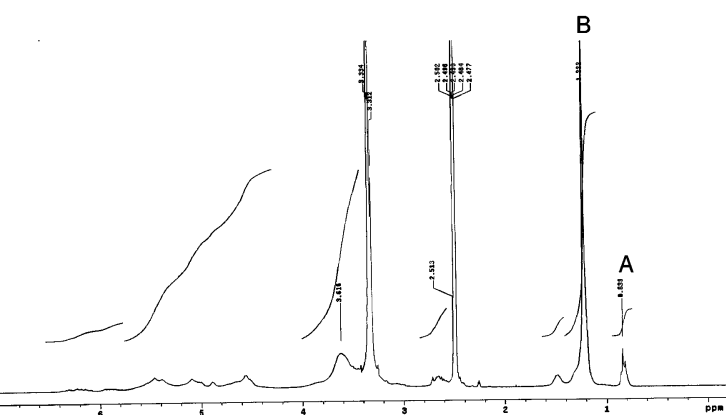

(a)

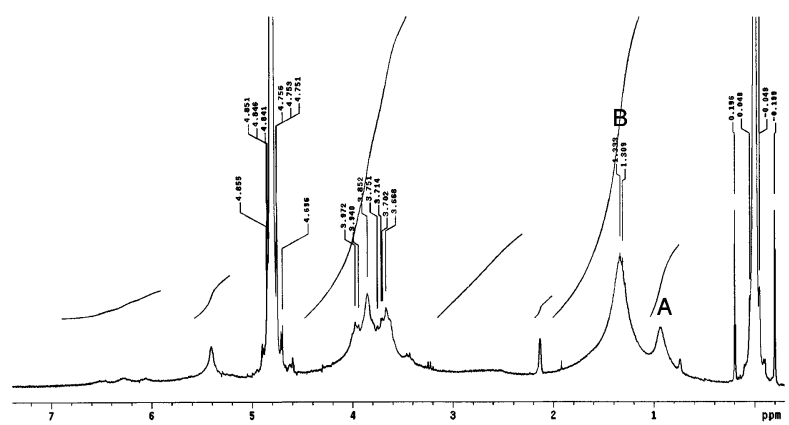

(c)

Figure 3. ${ }^{1} \mathrm{H}$ NMR spectra of $\operatorname{dex} C_{16}(10 \mathrm{mg} / \mathrm{mL})$ in (a) DMSO- $d_{6}$, (b) $10 \% D_{2} \mathrm{O}$ in DMSO- $d_{6}$, and (c) $\mathrm{D}_{2} \mathrm{O}$.

where $x$ is the average integral corresponding to the protons from alkyl moieties $(2.0-0.6 \mathrm{ppm})$ and $y$ is the integral of all dextrin protons $(3.0-5.8 \mathrm{ppm})$. Table 1 presents the DS values obtained for $\operatorname{dex}_{16}$ and the respective efficiency. The data presented in Table 1 correspond to the equilibrium position as it was verified by performing the assay with longer reaction times.

These results demonstrate that the synthesis method is versatile, allowing production, in a simple two-step procedure, of materials with controlled amounts of grafted hydrophobes. Thus, fine-tuning the properties of the materials is possible. Furthermore, different hydrophobic chemicals may be grafted (for instance, thiocholesterol).

Formation of Nanoparticles. The dissolution of $\operatorname{dex}_{16}$ in water is expected to give rise to micelle formation, owing to the amphiphilic nature of the molecule. The nanoparticles formation was accessed using both ${ }^{1} \mathrm{H}$ NMR and AFM.

Due to the limited mobility of the alkyl hydrophobic chains inside the nanoparticles, the shape and width of the ${ }^{1} \mathrm{H}$ NMR signals assigned to the alkyl chain protons $(2.0-0.6 \mathrm{ppm})$ are dependent on the solvent $\left(\mathrm{D}_{2} \mathrm{O} / \mathrm{DMSO}_{d}\right)$ used to record the ${ }^{1} \mathrm{H}$ NMR spectra. The ${ }^{1} \mathrm{H}$ NMR signals assigned to the methyl (0.8 ppm) and methylene (1.1 ppm) groups are sharp in DMSO (a good solvent for dex $\mathrm{C}_{16}$ ), but progressive broadening at the base is noticeable as the percentage of water in $\mathrm{D}_{2} \mathrm{O} / \mathrm{DMSO}$ mixtures increases. In pure $\mathrm{D}_{2} \mathrm{O}$ extensive broadening is noticeable (Figure $3 \mathrm{~b}$ and $\mathrm{c}$ ). The shape of the ${ }^{1} \mathrm{H}$ NMR alkyl chain signals in deuterated water is characteristic of a superposition of peaks representing a collection of chemically identical species yet possessing various degrees of mobility. ${ }^{11}$ This result suggests that alkyl chains have different environments when dispersed in water. Some chains might be involved in hydrophobic microdomains (low mobility) with others remaining exposed

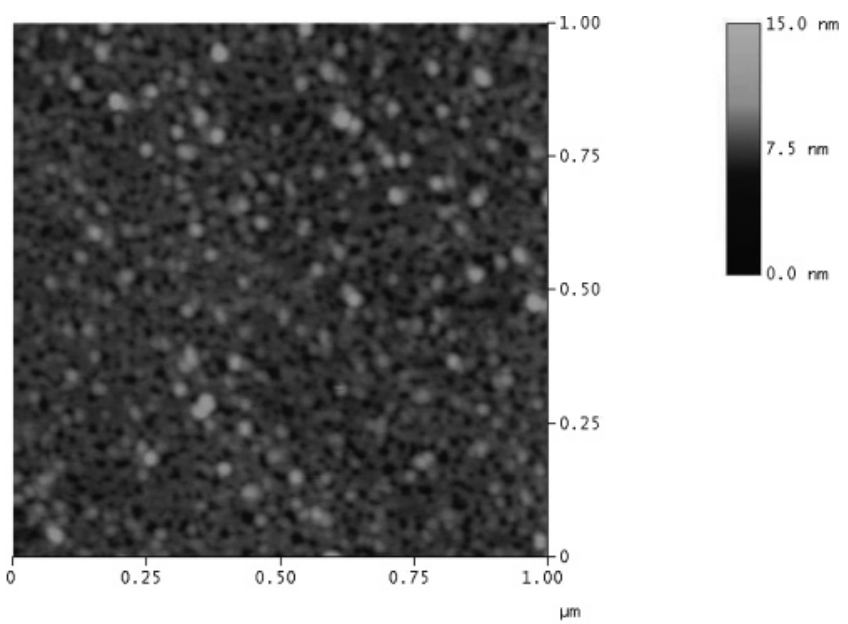

Figure 4. Tapping-mode AFM image of dex $\mathrm{C}_{16}\left(\mathrm{DS}_{\mathrm{C} 16} 7 \%\right)$ nanoparticles adsorbed on HOPG (or graphite) from aqueous solution (0.01 $\mathrm{g} / \mathrm{dL})$.

to the hydrophilic solvent (high mobility). In DMSO, all hydrophobic chains are exposed to the solvent, having the same mobility.

In addition, self-assembled particles were observed using AFM. The AFM images (Figure 4) reveal spherical nanoparticles. The AFM results indicate that, for $\operatorname{dex} \mathrm{C}_{16}$ with $\mathrm{DS}_{\mathrm{C} 16}$ $7 \%$, the mean diameter of nanoparticles is roughly $20 \mathrm{~nm}$. In general, the nanoparticles appear to have a narrow size distribution.

Nanoparticles Stability. To evaluate the stability of nanoparticles, the size was determined by DLS at $25^{\circ} \mathrm{C}$ up to 56 days (Figure 5).

Nanoparticles conserved their size during this study, indicating the high colloidal stability of nanoparticles in an aqueous medium. 


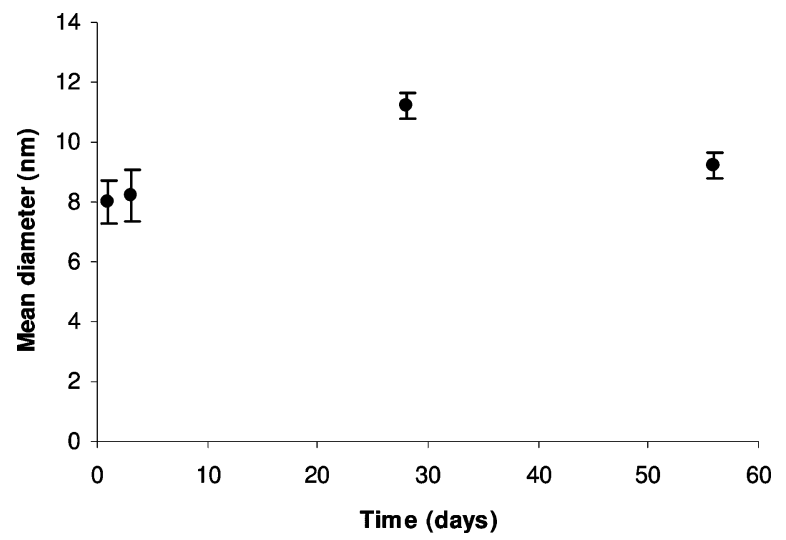

Figure 5. Colloidal stability of nanoparticles in water. The dex $\mathrm{C}_{16}$ (DS ${ }_{\mathrm{C} 16} 7 \%$ ) solution $(0.1 \mathrm{~g} / \mathrm{dL})$ was stored at $25{ }^{\circ} \mathrm{C}$ up to 56 days. The error bar is for standard deviation $(n=5)$.

\section{PDI $=0.684$, mean peak $=13.5 \mathrm{~nm} \%$ volume $=95.9 \%$}

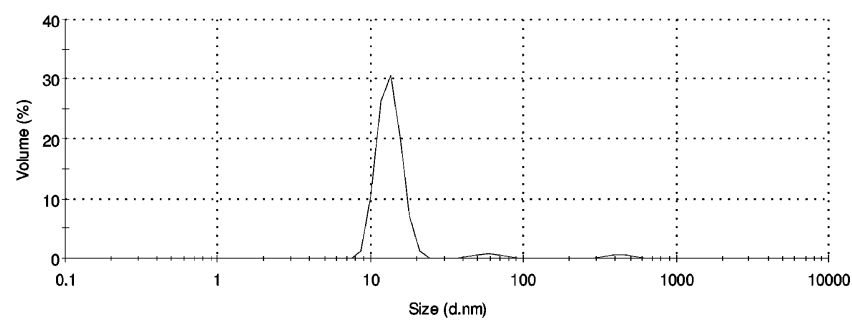

(a)

PDI $=0.651$, mean peak $=11,8 \mathrm{~nm} \%$ volume $=96.3 \%$

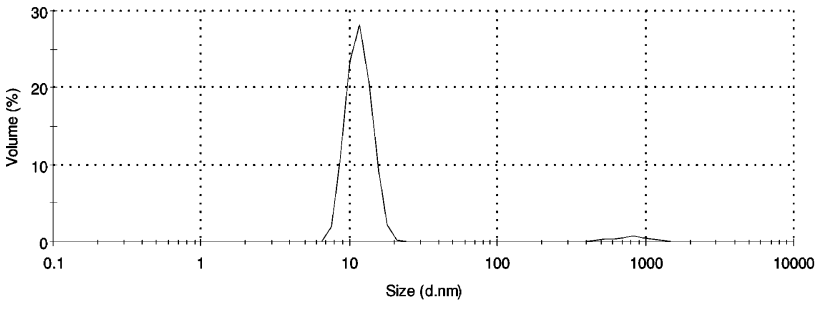

(b)

Figure 6. Size distribution of $\operatorname{dex} \mathrm{C}_{16}\left(\mathrm{DS}_{\mathrm{C} 16} 7 \%\right)$ aqueous dispersion (a) 0.01 and (b) $0.1 \mathrm{~g} / \mathrm{dL}$.

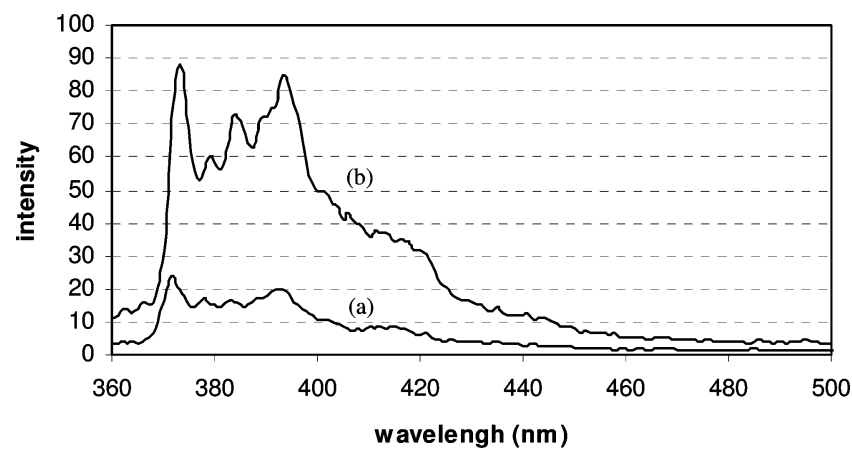

Figure 7. Emission spectra of (a) an aqueous solution of pyrene (1 $\left.\times 10^{-6} \mathrm{M}\right)$ and $(\mathrm{b})$ an aqueous solution of pyrene $\left(1 \times 10^{-6} \mathrm{M}\right)$ with dissolved dexC $\mathrm{C}_{16}\left(\mathrm{DS}_{\mathrm{C} 16} 7 \%\right)$ at a polymer concentration of $0.02 \mathrm{~g} / \mathrm{dL}$, $\lambda_{\mathrm{ex}}=337 \mathrm{~nm}$.

Size and Size Distribution. The dynamic light scattering (DLS) studies were done using a Nano-ZS (Malvern) instrument. Solutions with different concentrations of dexC $\mathrm{C}_{16}\left(\mathrm{DS}_{\mathrm{C} 16} 7 \%\right)$ were prepared by dilution of a $0.1 \mathrm{~g} / \mathrm{dL}$ solution and analyzed by DLS. The results are presented in Figure 6. DLS provides valuable information on the homogeneity of the dispersion. A single sharp peak in the DLS profile implies the existence of a

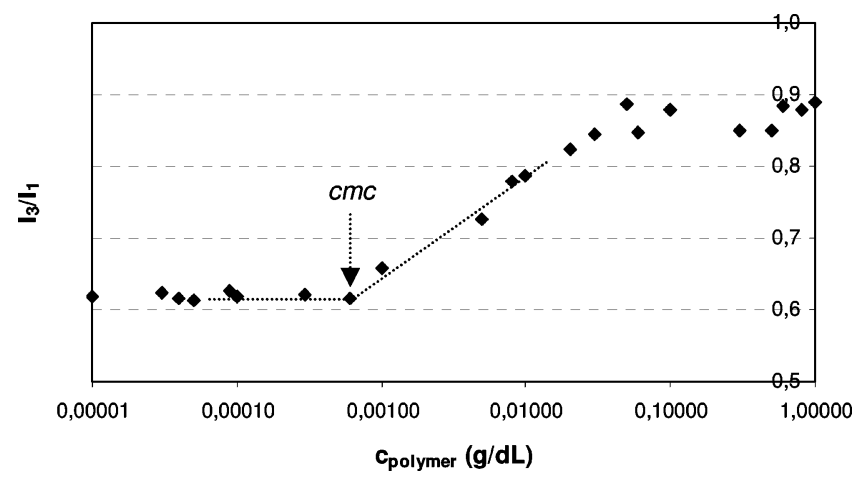

Figure 8. Fluorescence intensity ratio $I_{3} / l_{1}$ as a function of the $\operatorname{dex} \mathrm{C}_{16}$ (DS $\left.{ }_{116} 7 \%\right)$ concentration.

single population of particles. The polydispersity index (PdI) is also helpful in the characterization of the size distribution. PdI values close to 1 are indicative of heterogeneity.

Figure 6 shows that the dilution of the sample has an effect on the particle size in the concentration range used. The hydrodynamic diameter of the predominant population of nanoparticles increases slightly with decreasing concentration. The DLS results are in good agreement with AFM results presented above. The small nanoparticles with a diameter of around $10-15 \mathrm{~nm}$ are the predominant population.

Critical Micelle Concentration of Hydrophobized DextrinVA. Pyrene may be used as a fluorescent probe to detect aggregate formation. ${ }^{12}$ The fluorescent properties of pyrene change when it is transferred from an aqueous environment to the hydrophobic microenvironment of the nanoparticles. Figure 7 presents the fluorescence spectra of pyrene in water $(1 \times$ $\left.10^{-6} \mathrm{M}\right)$ and in the presence of dexC $\mathrm{C}_{16}$. The $\mathrm{cmc}$ was determined from the change in the ratio of intensities of the third and first peak $\left(I_{3} / I_{1}\right)$ in the emission spectra.

The pyrene concentration is in all experiments very low (1 $\times 10^{-6} \mathrm{M}$ ) to prevent excimer formation (detectable at $\approx 470$ $\mathrm{nm})$. The $I_{3} / I_{1}$ values, corresponding to samples with different polymer concentrations, were calculated and plotted in Figure 8.

At low polymer concentrations, $I_{3} / I_{1}$ is almost constant and equal to 0.62 , i.e., pyrene experiences an aqueous environment. For higher polymer concentrations $I_{3} / I_{1}$ starts to increase, showing that pyrene senses a less polar environment due to its solubilization in the hydrophobic domains that are formed by self-assembly (for $c_{\text {polymer }}>0.0008 \mathrm{~g} / \mathrm{dL}$ ), where it senses a less polar environment. The curve is sigmoidal, as observed for other amphiphilic polymers. The ratio $I_{3} / I_{1}$ eventually reaches a constant value as there are enough nanoparticles to solubilize all pyrene molecules. The variation in pyrene fluorescence is due to the partition between water and the hydrophobic microdomains until equilibrium (or probe saturation) occurs.

The cmc determination from fluorescence data can be based on different assumptions, due to the relatively large concentration range over which the fluorophore response stretches. Different researchers have proposed the $\mathrm{cmc}$ as either the onset, the offset, or the midpoint of the increased fluorophore response ratio as a function of the polymer concentration. ${ }^{12}$ In this study, the polymer concentration corresponding to the onset of the variation was considered the $\mathrm{cmc}$ as it indicates the onset of the association event (Figure 8). This event can be an incipient association of side-chain hydrophobes (at least the very first association of two hydrophobes). The results on Figure 8 


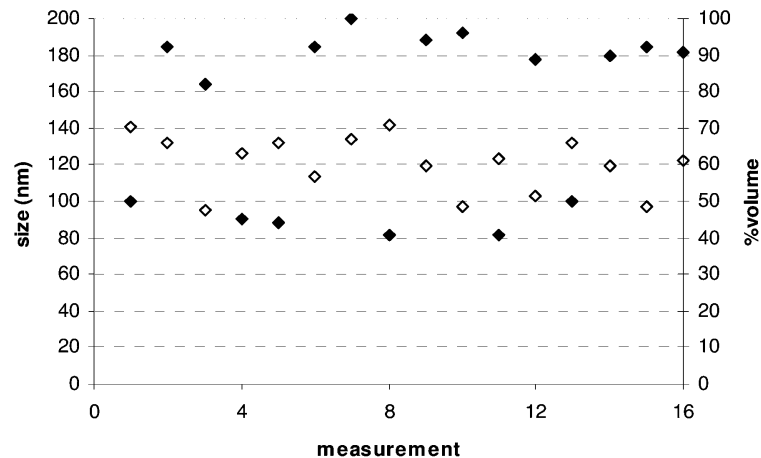

(a)

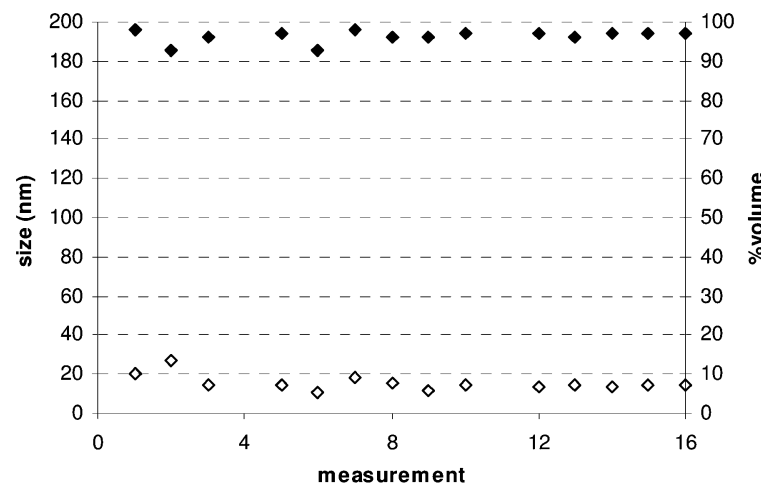

(c)

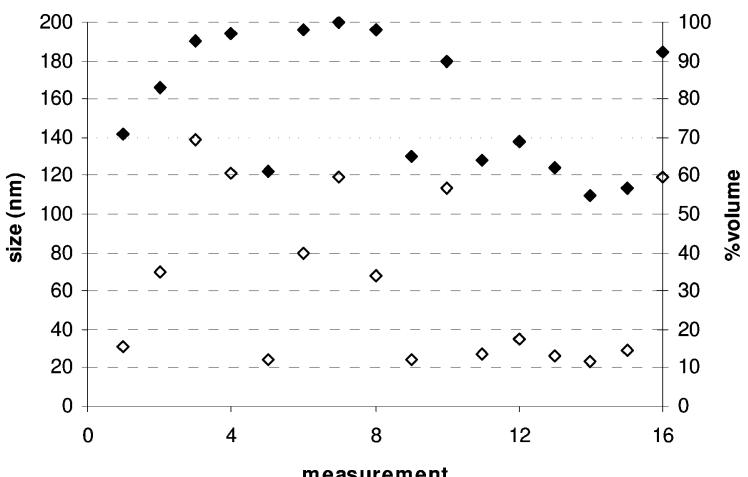

(b)

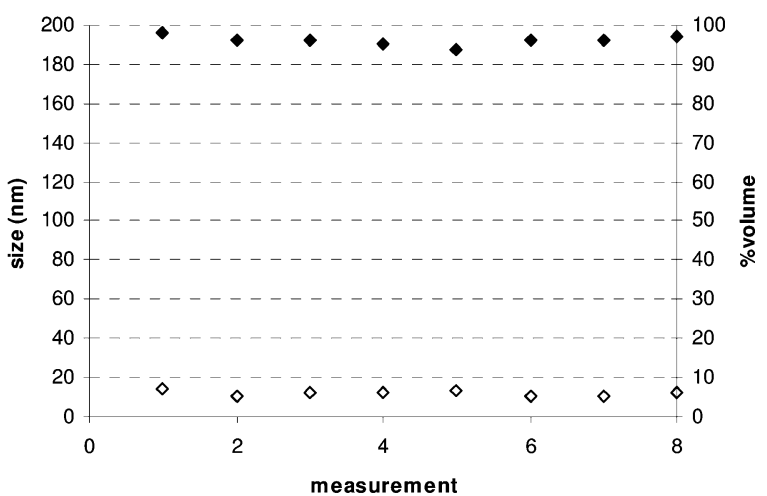

(d)

Figure 9. Size $(\diamond)$ and the respective percent volume $(\diamond)$ obtained in consecutive analysis for dexC ${ }_{16}\left(\mathrm{DS}_{\mathrm{C} 16} 7 \%\right)$ at (a) 0.0001 , (b) 0.001 , (c) 0.01 , and (d) $0.1 \mathrm{~g} / \mathrm{dL}$. While in experiments a, c, and d a consistent measure of the particles size was possible, for the concentration used in experiment $\mathrm{b}$, close to $\mathrm{cmc}$, the size detected varies from one measurement to another, suggesting that the material is unstable and particles with different size are present in the mixture.

demonstrate that a phase transition occurs when the polymer concentration increases ( $\mathrm{cmc}$ of about $0.0008 \mathrm{~g} / \mathrm{dL}$ ) owing to a structural reorganization of the dissolved molecules that leads to formation of hydrophobic domains. Dissolution of pyrene in these domains results in higher fluorescence intensity and $I_{3} / I_{1}$ values.

The variation in the particle sizes, as a function of the concentration, can also be studied by DLS. Figure 9 shows the particle size and the respective percent volume for $\operatorname{dex}_{16}\left(\mathrm{DS}_{\mathrm{C} 16}\right.$ $7 \%$ ) at polymer concentrations ranging between 0.0001 and $0.1 \mathrm{~g} / \mathrm{dL}$.

The particle size detected depends on the sample concentration, a dramatic reduction in size being detected as the concentration reaches cmc (as roughly estimated by DLS and showing good agreement with the fluorescence experiments). The particles have a hydrodynamic diameter inferior to $20 \mathrm{~nm}$ for the higher concentrations (Figure 9c and d) and a hydrodynamic diameter of about $120 \mathrm{~nm}$ for the lower concentrations (Figure 9a). A transition is noticeable at about $0.001 \mathrm{~g} / \mathrm{dL}$ (Figure 9b). Thus, it appears that the polymer is not very soluble, and probably aggregates are being detected in the DLS measurements for the low polymer concentration. In fact, dextrin-obtained from starch-is not very soluble, giving rise to colloidal dispersions in water. As the concentration increases, the hydrophobic domains give rise to formation of nanoparticles with a hydrophobic core (as shown in the fluorescence experiments). Apparently, nanoparticle formation gives rise to a more compact structure induced by the hydrophobic force. The beginning of the association event seems to occur close to 0.001 $\mathrm{g} / \mathrm{dL}$, corresponding to the $\mathrm{cmc}$, in good agreement with the fluorescence results.

\section{Conclusions}

In the present study we report the synthesis of a hydrophobized dextrin polymer, dexC $\mathrm{C}_{16}$. The synthesis method is versatile as it allows controlling the degree of substitution with hexadecanethiol and therefore fine-tuning the properties of the materials. DexC $\mathrm{C}_{16}$ self-aggregates in water, originating colloidally stable (over 2 months) nanoparticles with a narrow size distribution. A diameter of about $20 \mathrm{~nm}$ was determined by DLS and AFM. The cmc value, around $0.001 \mathrm{~g} / \mathrm{dL}$, was determined using pyrene as a fluorescent probe and confirmed by DLS. The value determined is lower than that reported for the most common low molecular weight surfactants. The low cmc may be due to the low solubility of dextrin. Reaction of dextrin-VA with other (thiol) nucleophiles might open access to a variety of amphiphilic materials with tailored properties.

Acknowledgment. The authors acknowledge the contributions from Dr. Lino Ferreira for fruitful discussions and Drs. Margarida Bastos, Marieta Nichifor, and Paula Gameiro for revising the manuscript and contributions to the fluorescence analysis.

\section{References and Notes}

(1) Akiyoshi, K.; Deguchi, S.; Moriguchi, N.; Yamaguchi, S.; Sunamoto, J. Macromolecules 1993, 26, 3062-3068.

(2) Akiyoshi, K.; Kobayashi, S.; Shichibe, S.; Mix, D.; Baudys, M.; Kim, S. W.; Sunamoto, J. J. Controlled Release 1998, 54, 313-320.

(3) Liu, C.; Chen, X.; Park, H. Carbohydr. Polym. 2005, 62, 293-298.

(4) Akiyoshi, K.; Sunamoto, J. Supramol. Sci. 1996, 3, 157-163.

(5) Petit-Agnely, F.; Iliopoulos, I.; Zana, R. Langmuir 2000, 16, 99219927.

(6) Petit-Agnely, F.; Iliopoulos, I. J. Phys. Chem. B 1999, 103, 48034808. 
(7) Lee, I.; Akiyoshi, K. Biomaterials 2004, 25, 2911-2918.

(8) Hans, M.; Shimoni, K.; Danino, D.; Siegel, S. J.; Lowman, A. Biomacromolecules 6, 2708-2717.

(9) Nizri, G.; Magdassi, S. J. Colloid Interface Sci. 2005, 291, 169174.

(10) Ferreira, L.; Carvalho, R.; Gil, M. H.; Dordick, J. S. Biomaterials 2002, 23, 3957-3967.
(11) Hrkach, J. S.; Peracchia, M. T.; Domb, A.; Lotan, N.; Langer, R. Biomaterials 1997, 18, 27-30.

(12) Nichifor, M.; Lopes, S.; Bastos, M.; Lopes, A. J. Phys. Chem. B 2004, 108, 16463-16472.

BM060993E 